\title{
Multi-Stage Pedestrian Crossings and Two-Stage Bicycle Turns: Delay Estimation and Signal Timing Techniques for Limiting Pedestrian and Bicycle Delay
}

\author{
Peter G. Furth*, Yue (Danny) Wang, Michael A. Santos \\ Northeastern University, Boston, MA, USA \\ Email: *p.furth@neu.edu, wang.yue3@husky.neu.edu, santos.michael.a@gmail.com
}

How to cite this paper: Furth, P.G., Wang, Y. and Santos, M.A. (2019) Multi-Stage Pedestrian Crossings and Two-Stage Bicycle Turns: Delay Estimation and Signal Timing Techniques for Limiting Pedestrian and Bicycle Delay. Journal of Transportation Technologies, 9, 489-503. https://doi.org/10.4236/jtts.2019.94031

Received: November 20, 2018

Accepted: October 7, 2019

Published: October 10, 2019

Copyright (อ 2019 by author(s) and Scientific Research Publishing Inc. This work is licensed under the Creative Commons Attribution International License (CC BY 4.0).

http://creativecommons.org/licenses/by/4.0/

\section{(c) (i) Open Access}

\begin{abstract}
Signalized intersections sometimes involve multistage pedestrian crossings, in which pedestrians cross to one or more islands and then wait there for a signal to continue. When signals are timed without attention to pedestrian progression, pedestrian delay at multistage crossings can be very long. This paper addresses two issues. First, pedestrian delay at multistage crossings is rarely evaluated because there are no tools in the industry for that purpose except microsimulation. We present a numerical method for determining crossing delay with any number of stages and with the possibility of multiple WALK intervals per cycle. The same method can be applied to single stage crossings, to diagonal two-stage crossings where pedestrians may have path choice, and bicycle two-stage turns. This method has been implemented in a freely available online tool. Second, we describe several signal timing techniques for improving pedestrian and bicyclist progression, and thus reducing their delay, through multistage crossings. They include reservice for selected crossing phases, left turn overlaps, having pedestrian phases overlap each other, and bidirectional bicycle crossings which create path options for two-stage turns. Examples show the potential for large reductions in pedestrian delay, often with little or no increase in vehicular delay. In one example, the addition of a short pedestrian overlap phase reduced average pedestrian delay at a 3-stage crossing by $82 \mathrm{~s}$ while average vehicular delay increased by only $0.5 \mathrm{~s}$.
\end{abstract}

\section{Keywords}

Pedestrian Delay, Traffic Signal Control, Multistage Crossings

\section{Introduction}

Signalized intersections sometimes involve multistage crossings in which pede- 
strians cross to an island and are expected to wait there for a signal there to resume crossing. Two-stage crossings are common; three- and four-stage crossings are also used.

While multistage crossings can improve an intersection's efficiency, they can also lead to large pedestrian delays if pedestrian progression through the different stages is poor. While the Manual on Uniform Traffic Control Devices [1] specifies that all of the safety provisions that apply to signalized crosswalks must be applied to each stage of a multistage crossing individually, it is silent on whether or how those stages are to be coordinated. Unfortunately, in current practice, there are no easily applied tools for evaluating pedestrian delay at multistage crossings. Using standard formulas for pedestrian delay at each stage and adding them is incorrect, because those formulas assume random arrivals, and pedestrian arrivals at every stage after the first is not at all random. Because of the lack of analysis methods, intersection designs with multistage crossings are routinely advanced and implemented without any evaluation of pedestrian progression or delay. No wonder then that pedestrian delay is often very long.

For bicyclists, two-stage left turns [2] are a form of multistage crossing. A cyclist turning left may do so in a "vehicular manner", i.e. using the same left turn lane as cars, in a single stage; however, more and more bike lanes-particularly protected bike lanes-are designed with the idea that cyclists will turn left in two stages, as a pedestrian would. Again, standard methods of delay evaluation do not apply, and methods are needed to ensure that cyclist delay is properly measured.

Part 1 of this paper describes a method for evaluating pedestrian and bicyclist delay at multistage crossings. This method has been implemented in a freely available software tool. Part 2 describes signal timing techniques for achieving good pedestrian progression through multistage crossings and thus limiting pedestrian delay. Examples show that by using these techniques, intersections with multistage crossings can offer pedestrians a good level of service while retaining their efficiency advantages for vehicular traffic.

\section{Estimating Multistage Crossing Delay}

\section{Existing Methods for Estimating Crossing Delay}

At single stage crossings, average pedestrian delay can be evaluated rather easily. Except where pedestrian volumes are so great that pedestrians queue up several people deep, pedestrians can be assumed to cross without interference from other pedestrians, making pedestrian counts unnecessary for estimating delay. The signal cycle can be divided into two intervals: effective pedestrian green, in which pedestrians are allowed to start crossing, and the rest of the cycle, called effective pedestrian red. Effective pedestrian green consists of the WALK interval plus the first few seconds following it. Legally, pedestrians are supposed to begin crossing only during the WALK interval, but observation indicates that most pedestrians are still willing to begin crossing a few seconds after it ends. The Highway Capacity Manual (HCM) [3] suggests $4 \mathrm{~s}$ as a default for this extra 
walk time, based on research done by Pretty in the 1970s [4].

The well-accepted Formula (1) for average pedestrian delay $\left(d_{p e d}\right)$ for a single-stage crossing is

$$
d_{p e d}=\left(\frac{r_{p e d}}{C}\right)\left(\frac{r_{p e d}}{2}\right)
$$

where $C=$ cycle length and $r_{p e d}=$ length of effective pedestrian red [3]. It is based on the assumption that pedestrians are equally likely to arrive at any moment in the cycle (uniform arrivals). The first ratio is the fraction of pedestrians arriving on red, and the second is the average delay to those that do arrive on red. Those who arrive during pedestrian green, of course, have no delay.

With multistage crossings, it would be wrong to evaluate pedestrian delay by simply applying Equation (1) to each crossing stage and summing, because at every stage except the first, pedestrians do not arrive uniformly; instead, they arrive in platoons as released by the previous stage's signal [5]. Wang and Tian [6] and $\mathrm{Ma}$ et al. [7] derived formulas to evaluate delay at a two-stage crossing. Wang and Tian developed formulas for six different cases, and Ma et al. for four, depending on which signal (green or red) pedestrians see when arriving at the first and second stages. Both methods involve averaging results over all possible cases using appropriate weights. Because of their complexity, these formulas have not seen widespread adoption. Furthermore, the logic they follow is such that complexity greatly expands when important generalizations are considered, including three that this paper considers-crossing with three or more stages, two or more WALK intervals per cycle, and multistage crossings in which pedestrians have a choice of which path to follow.

Microsimulation software that explicitly models pedestrian movements can be used to evaluate pedestrian delay at multistage crossings. However, microsimulation is beyond the scope of most intersection design projects because of the time and expertise needed.

There remains therefore a need for a simple method for calculating average pedestrian delay at multi-stage crossings, one that can be applied as easily as those used to calculate average delay and level of service for motor traffic.

\section{A Numerical Method for Evaluating Multistage Crossing Delay}

Instead of using analytical formulas, we propose calculating pedestrian delay numerically. A signal cycle is divided into a large number of time steps; to limit approximation error, steps of $0.1 \mathrm{~s}$ are recommended. Pedestrians are assumed to arrive with equal likelihood during every time step. For each arrival moment, a pedestrian's trajectory can be traced through the crossing, with the pedestrian waiting or walking according to the signal indication until finishing the last crossing. For any given trajectory, delay equals time in the system minus time spent walking. In one sense, this approach is a simulation, because one pedestrian for each arrival moment (each time step) is tracked through the intersection, with results averaged over all possible arrival moments. However, unlike microsimulation, the method is deterministic. 
This method is readily expandable to any number of stages, any number of walk intervals per cycle, and path choice. It is computationally fast. It lends itself well to graphical representation, making it easy to understand, easy to check for errors, and easy to explain to the public.

Tracing a pedestrian trajectory for a given initial arrival time involves the following steps. Assume the crossing has $n$ stages. Initial arrival time, $t_{i n}$, is given. Let $t_{\text {arr }}$ and $t_{\text {dep }}$ denote arrival time and departure time, respectively, at the current stage.

1) Initialize. Crossing Stage $=1 . t_{\text {arr }}=t_{\text {in }}$. Accumulated Walk $=0$.

2) Departure time. If the signal state for Crossing Stage at time $t_{\text {arr }}$ is effective green, $t_{d e p}=t_{\text {arr }}$ Otherwise, $t_{d e p}=$ next moment at which the signal state becomes effective green.

3) If Crossing Stage $<n$, advance to the next step. Otherwise, set $t_{\text {out }}=t_{\text {dep }}$; calculate Time in System $=t_{\text {out }}-t_{i n}$; calculate delay $=$ Time in System-Accumulated Walk. STOP.

4) Walking time. $t_{\text {walk }}=$ distance to the next stage/Walk Speed; augment $A c$ cumulated Walk by $t_{\text {walk }}$. Distance to the next stage is the crossing length for Crossing Stage plus the distance across the island to the stopline for the next crossing stage. A standard walking speed such as $4.5 \mathrm{ft} / \mathrm{s}$, intended to represent an average walking speed, can be applied. (It is also possible to use a distribution of walking speeds to account for a mix of faster and slower pedestrians. We tested this approach and found that it yielded no meaningful difference in results compared to using average walking speed).

5) Advance to the next stage. $t_{\text {arr }}=t_{\text {dep }}+t_{\text {walk }}$ Augment Crossing Stage by 1.Go to step 2.

We have programmed this method using MatLab as the computational engine. It is freely available for download as the Northeastern University Ped \& Bike Crossing Delay Calculator [8]. (Using the program does not require a MatLab license). It has one module for sequential crossings and another for diagonal crossings that may involve path choice. Users enter walk distances (i.e. crosswalk lengths and island widths) and signal timing data (start time and duration of each walk interval). User-adjustable parameters are walking speed (default $=4.5$ $\mathrm{ft} / \mathrm{s}$ ) and extra time after the WALK interval that is still considered effective green (default $=4 \mathrm{~s}$ ). The output is average delay at each stage of the crossing and overall, and a progression diagram with trajectories illustrating pedestrian progression. Pedestrian volume data is not needed, because of the assumption that pedestrians will not be substantially delayed by each other.

Figure 1 offers an example of this numerical analysis method. The site is in Boston, where the Muddy River Path crosses Riverway at its intersection with Park Drive. Pedestrians have a 3-stage crossing, labeled ABCD in Figure 1. Pertinent data, including the cycle length (100 s), start time and duration of each WALK interval, and walking distances are shown in the figure. The progression diagram, in which thick horizontal lines indicate an accumulation of pedestrians waiting, shows how poor the progression is. Pedestrians going in direction 


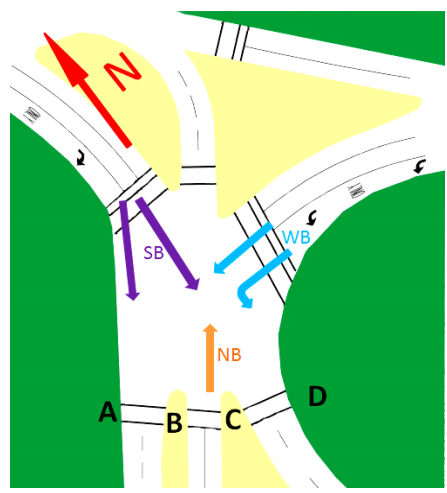

(a)

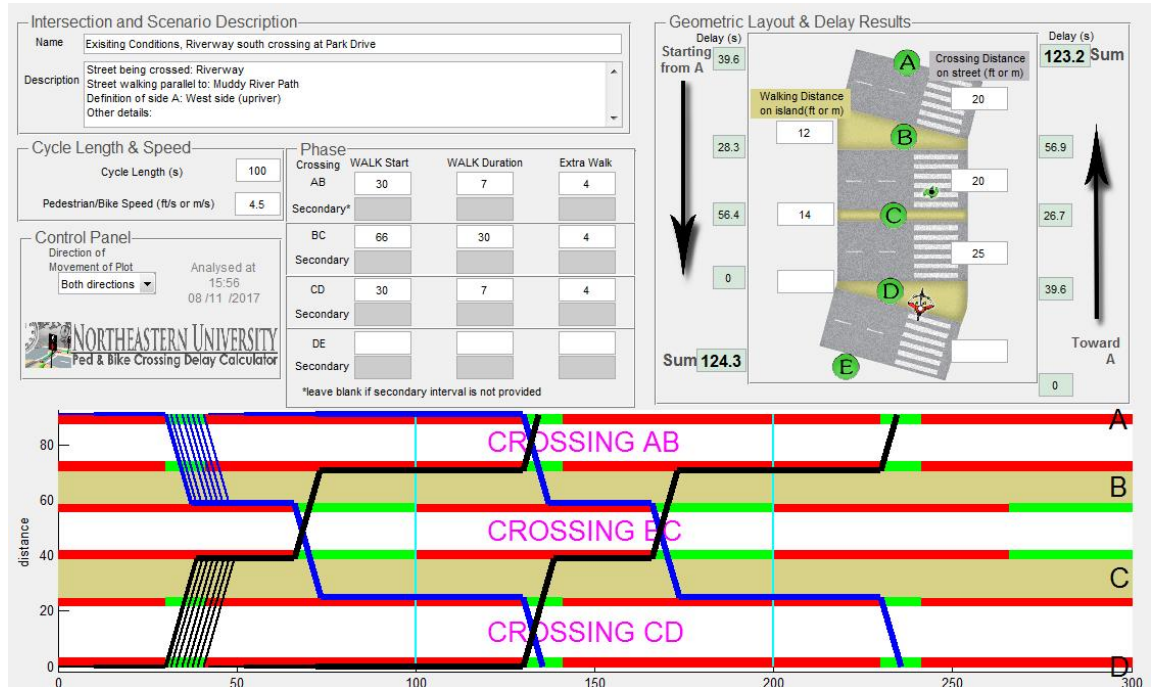

(b)

Figure 1. Three stage crossing for the Muddy River Path across Riverway, Boston, MA. (a) Layout; (b) Delay calculation and progression graphics.

$\mathrm{ABCD}$ accumulate while waiting a long time at A until there's a WALK signal, and then walk in a platoon to island B; there, they wait almost $30 \mathrm{~s}$, and then travel en masse to island C, where they wait roughly $60 \mathrm{~s}$ for a WALK to finish their crossing. Overall, average delay is $124 \mathrm{~s}$ in direction $\mathrm{ABCD}$, and $123 \mathrm{~s}$ in direction DCBA-more than double the 60 s limit used in the Highway Capacity Manual to define level of service " $F$ ", the worst possible level of service.

It is highly unlikely that this timing plan, implemented in 2016, would have been approved if these average delays had been calculated and reported to the public and to approving agencies. Later in this paper, we show that with a modification to the timing plan, pedestrian delay can be reduced drastically with no discernable impact on vehicular delay.

To confirm the proposed method, the two-stage crossing described by Wang and Tian [6], crossing Boulder Highway at Flamingo Road in Las Vegas, was evaluated. They found the delay to be $55.8 \mathrm{~s}$ in each direction; our method finds the average delay to be $55.8 \mathrm{~s}$ eastbound and $55.9 \mathrm{~s}$ westbound. The $0.1 \mathrm{~s}$ discrepancy is approximation error due to using finite time steps. Confirmation was 
also obtained by comparing the model's Riverway crossing results with results from VISSIM, a microsimulation software that explicitly models pedestrians. Average delay calculated by VISSIM was $120 \mathrm{~s}$ in one direction and $122 \mathrm{~s}$ in the other, versus $123 \mathrm{~s}$ and $124 \mathrm{~s}$ using the numerical method. The discrepancies are within the margin of error expected for a stochastic simulation.

\section{Evaluating Delay at Intersections with Actuated Control}

Our method, like those cited in the literature, treats cycle length and pedestrian interval timing as given, while many intersections use actuated control logic in which the length of relevant intervals is not fixed. For isolated actuated signals, expected values of cycle length and phase lengths can be estimated following the methods described in the HCM or in Furth, Cesme, and Muller [9]. For coordinated-actuated signals, cycle length is fixed, but analysts may have to estimate the start time and duration of WALK intervals. During peak periods, for which evaluation is often most critical, actual operations often match nominal timings closely as phases tend to run to their maximum split. Where flexible control logic makes the signal operation highly variable, microsimulation may be the only reliable way to estimate performance.

\section{Estimating Delay for Diagonal Crossings with Path Choice}

Pedestrians crossing to a diagonally opposite corner of an intersection normally make their crossing in two stages, passing through another corner en route. Where such a movement is important for transportation planning-for example, for a major crossing from a railroad station-designers will be interested in estimating and limiting the delay for this 2-stage movement.

Diagonal crossings usually involve path choice. If the corners of an intersection are labeled clockwise A-B-C-D, then a pedestrian crossing from $A$ to $C$ will typically have the choice of path A-B-C (clockwise) or A-D-C (counterclockwise). Where there is no choice-say, because one of the crossings is closed-a diagonal crossing can be treated as a sequential multistage crossing. But where there is path choice, additional logic is needed.

We assume that pedestrians identify the feasible paths and take whichever path offers a WALK signal first. With this assumption, there is a unique chosen path for every arrival moment, so that trajectories for an arrival in each time step can readily be constructed and delay calculated, as before. In the unlikely event that both paths offer WALK at the same time at the first stage, demand is split equally between the paths.

An example calculation is given in Figure 2 for a hypothetical intersection with 8-phase, dual ring control, leading lefts, concurrent crossings, and an $80 \mathrm{~s}$ cycle. Other relevant timing parameters are shown in the figure. As the figure shows, with path choice, average delay from corner A to corner C is $33 \mathrm{~s}$. Another calculation (not shown) finds that if pedestrians were restricted to a single path, average delay would instead be $53 \mathrm{~s}$ for path A-B-C and $52 \mathrm{~s}$ for path A-D-C.

\section{Bicyclist Two-Stage Left Turns}

Typically, marked bicycle crossings, sometimes called cross bikes or cross 


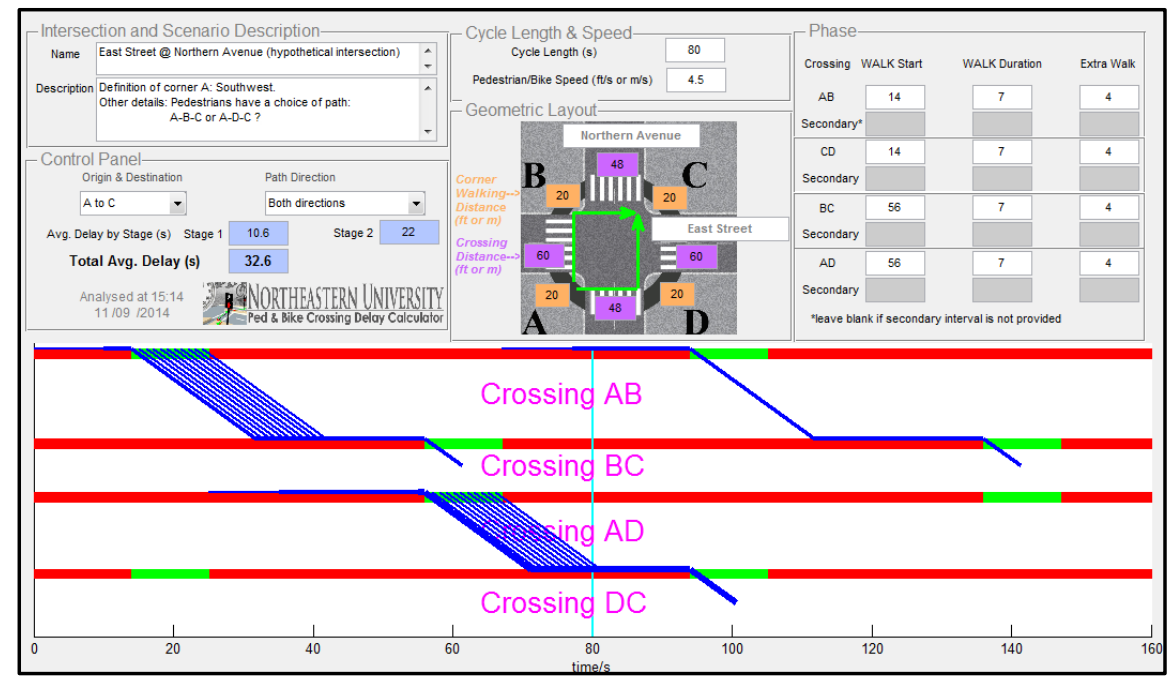

Figure 2. Delay calculation and progression graphics for a diagonal crossing. Pedestrians are crossing from $\mathrm{A}$ to $\mathrm{C}$, and may travel via $\mathrm{B}$ or via $\mathrm{D}$.

rides, are unidirectional, in which case a cyclist making a two-stage left turn has only one legal path. In such a case, cyclist delay for the left turn movement can be calculated by treating it as a sequential two-stage crossing. However, if bicycle crossings are marked as bidirectional-a tactic recommended in the Dutch bikeway manual [10] - cyclists can take advantage of path choice, just like pedestrians making a diagonal crossing, thereby reducing their delay.

Using the same example shown in Figure 2, but with cyclist speed set to 12 $\mathrm{ft} / \mathrm{s}$ and cyclists allowed to start through the entire vehicular green, their average delay from corner $\mathrm{A}$ to corner $\mathrm{C}$ will be $42 \mathrm{~s}$ if crossings are bidirectional, versus $64 \mathrm{~s}$ if crossings are unidirectional.

It is interesting to compare these results with delay for a vehicular left-turn, made in a single stage from the left turn lane. If overflow delay can be ignored, the HCM s uniform delay formula may be used to estimate delay [3]. Assuming that the left turn phase has $10 \mathrm{~s}$ of effective green per cycle and a volume-capacity ratio of 0.8 , average delay for a single-stage crossing will be $38 \mathrm{~s}$. In this example, then, providing bidirectional crossings reduces two-stage turning delay to the point that it is comparable to delay associated with a vehicular-style, single-stage left turn.

\section{Signal Timing Techniques for Limiting Delay at Multi-Stage Crossings}

Just as traffic signals along an arterial can be timed to offer good progression for vehicles, they can also be timed to offer good progression to pedestrians passing through multiple crossing stages. This section describes several signal timing techniques that can be used to improve pedestrian progression.

\section{Technique 1: Offset Crossing Phases with Short Cycles}

For crossing a wide street with a median, a single-stage crossing demands a long crossing phase, reducing traffic capacity. To limit that traffic capacity im- 
pact, the crossing can be configured as a pair of half-crossings, timed with only enough pedestrian clearance time to reach the end of the half-crossing, forcing pedestrians to wait in the median for the next WALK phase. If the WALK phases for the two half-crossings are simultaneous, pedestrians will have to wait almost a full cycle to cross, and if the cycle is long, the average delay will be quite large.

The first way to lower this delay is to reduce the cycle length. Because a half-crossing requires a shorter phase than a full crossing, it should be possible to provide the same vehicular capacity with a shorter cycle. A second way, relevant mainly at mid-block crossings, is to offset the two crossing phases by half a cycle. Pedestrians will have to wait in the median after the first half-crossing, but only for a limited time because the second crossing stage begins only half a cycle after the first stage. If the cycle is short, the wait in the middle can be very short.

The crossing of Huntington Avenue at Opera Place in Boston offers an illustrative example (see Figure 3(a)). Vehicular demand on Opera Place is so little that, if run concurrently with the parallel crossing, it can be served in the time needed for pedestrians, so that in effect, the intersection can be timed as if it were a midblock crossing. Huntington Ave. is part of an arterial coordination scheme whose cycle length in the p.m. peak is $100 \mathrm{~s}$.

Three alternatives can be compared: 1) a single stage crossing with a $100 \mathrm{~s}$ cycle, 2) a two-stage crossing with a $100 \mathrm{~s}$ cycle (the existing timing), and 3) a two-stage crossing with a $50 \mathrm{~s}$ cycle ("double cycling"), with the crossing phases

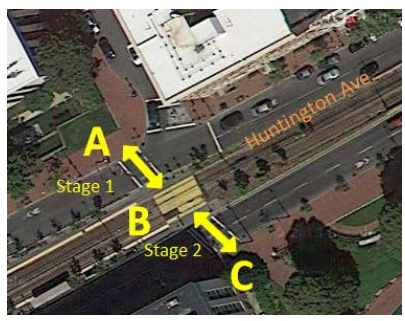

(a)

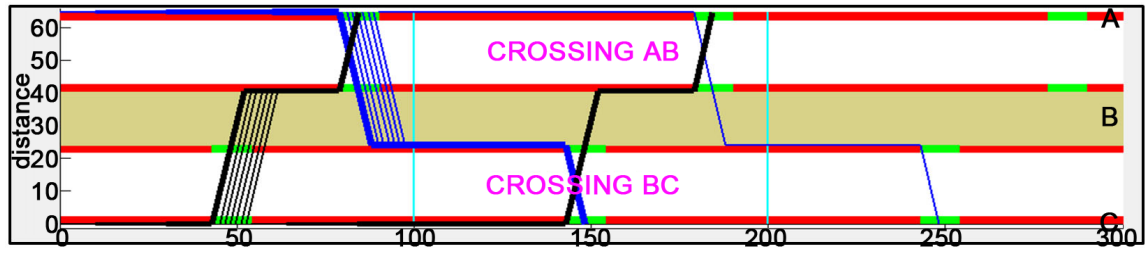

(b)

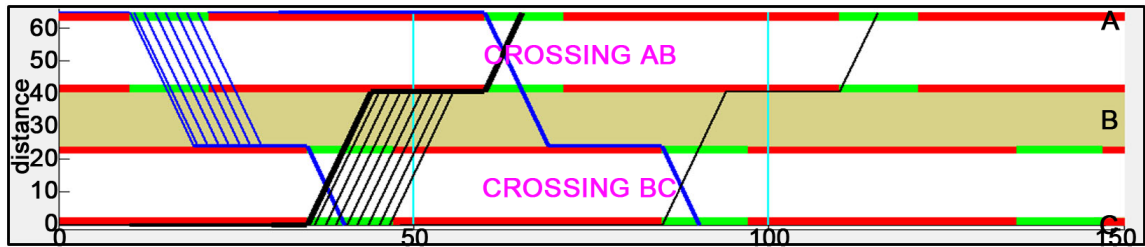

(c)

Figure 3. Two-stage crossing trajectories, Huntington Ave @ Opera Pl, Boston. (a) Layout; (b) Existing timing plan (b) with $100 \mathrm{~s}$ cycle; (c) Proposed timing plan (c) $50 \mathrm{~s}$ cycle, with crossing phases offset by $25 \mathrm{~s}$. 
offset from each other by $25 \mathrm{~s}$. Using a WALK window of $7 \mathrm{~s}$ and pedestrian speed of $3.5 \mathrm{ft} / \mathrm{s}$ for clearance, the length of the pedestrian phase is $27 \mathrm{~s}$ for a single stage crossing and $15 \mathrm{~s}$ with two-stage crossings. Figure 3(b) and Figure 3(c) show pedestrian progression for the two-stage crossing alternatives. For plan (2), one can see how long pedestrians have to wait, both for the initial crossing and again in the median, while for plan (3), one can see how much shorter are the waits. Table 1 shows average pedestrian delay and, as a measure of vehicular capacity, green ratio (for the roadway) for the three alternatives. Compared to a single stage crossing, using two-stage crossings with the cycle length kept at 100 $\mathrm{s}$ results in an average pedestrian delay of $79 \mathrm{~s}$, which is unacceptably large to most pedestrians (any observer at this intersection will see pedestrians routinely crossing against the light rather than wait that long). However, when combined with a low cycle length and clever offset, the two-stage crossing offers the least pedestrian delay of the three alternatives, only $30 \mathrm{~s}$. What's more, compared to a single stage crossing, double cycling with two-stage crossings lowers vehicular capacity by only $10 \%$, from a green ratio of 0.69 to 0.62 .

\section{Technique 2: Reservice for Short Crossings}

Consider a crossing across a channelized right turn lane. While such crossings are often unsignalized, they are sometimes signalized for safety reasons, e.g. because the intersection geometry allows the turn to be made at high speed. Such a crossing is short, and the right turn lane typically has excess capacity because it has few conflicts. Those conditions often make it possible to provide two or even more WALK intervals per cycle ("reservice"). Even if other crossings in the intersection offer only one WALK interval per cycle, reservice for the right-lane crossing(s) can vastly improve pedestrian progression.

To illustrate, consider the Boston intersection of Charlesgate with Boylston Street (Figure 4), with 3-stage crossing A-B-C-D. Crossing AB crosses a channelized right turn lane with no other conflicts. Figure 4 shows the progression for the existing timing, with long waits evident, and for an alternative with two WALK intervals per cycle for crossing $\mathrm{AB}$. Current average pedestrian delay for the three-stage crossing is $68 \mathrm{~s}$ southbound and $41 \mathrm{~s}$ northbound. With reservice for crossing $\mathrm{AB}$, pedestrian delay falls to $36 \mathrm{~s}$ southbound and $33 \mathrm{~s}$ northbound, and at the same time, analysis with vehicular volumes (not shown) indicates that vehicular delay for the right turns slightly decreases, because its current long red interval is replaced with two short red intervals.

Table 1. Pedestrian delay and vehicular capacity impacts of two-stage crossing with short cycle compared to other alternatives.

\begin{tabular}{lcc}
\hline \multicolumn{1}{c}{ Alternative } & $\begin{array}{c}\text { Average } \\
\text { pedestrian delay (s) }\end{array}$ & $\begin{array}{c}\text { Vehicular } \\
\text { green ratio }\end{array}$ \\
\hline 1) Single-stage crossing, 100 s cycle & 39 & 0.69 \\
2) Two-stage crossing, 100 s cycle (existing) & 79 & 0.81 \\
3) Two-stage crossing, 50 s cycle, offset crossing phases & 30 & 0.62 \\
\hline
\end{tabular}




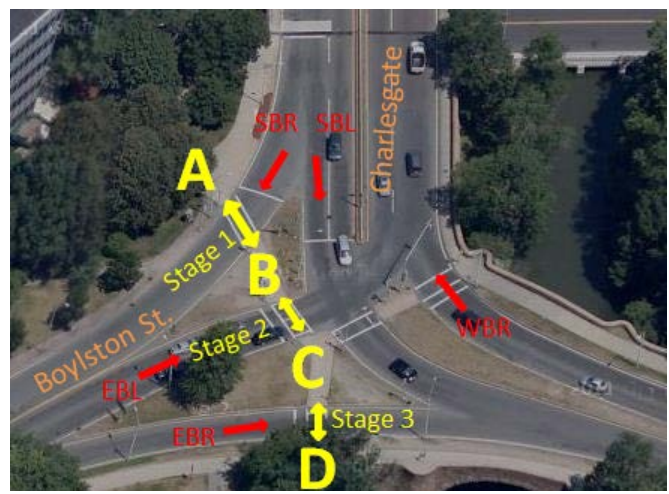

(a)

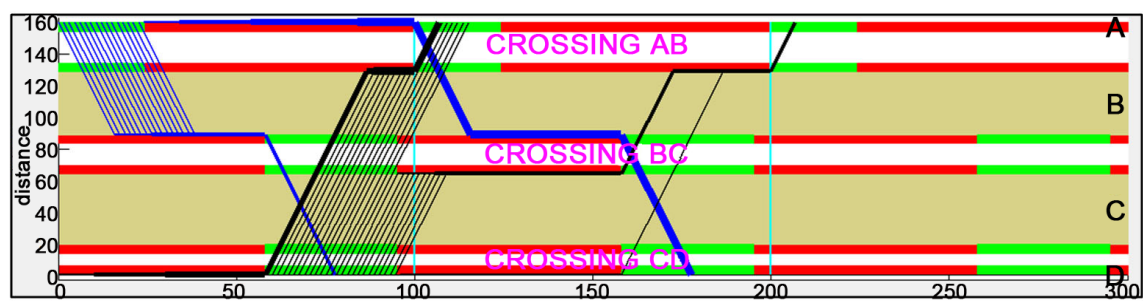

(b)

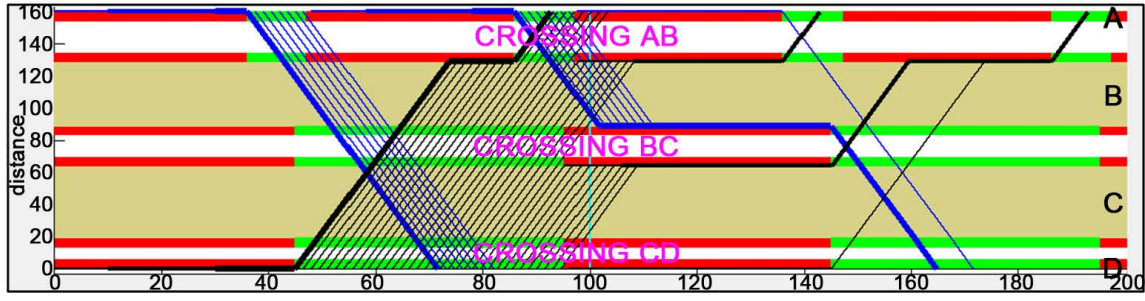

(c)

Figure 4. Reservice for one stage of a three-stage crossing across Boylston Street at Charlesgate, Boston. (a) Layout; (b) Timing plan and crossing trajectories, existing (C=100 s); (c) Timing plan and crossing trajectories, with pedestrian reservice in Stage $1(\mathrm{C}=100 \mathrm{~s})$.

At a 4-leg intersection with channelized right turns, pedestrians have to make 3-stage crossings - a short crossing to a delta island, then a main crossing to another delta island, and then another short crossing. If the short crossings across the right turn lanes are signalized, then providing reservice is critical to having good progression, since pedestrians crossing to any given delta island may be continuing along either the north-south or east-west axis, and with only a single WALK interval per cycle, it is impossible to provide good progression for crossings along both axes.

\section{Technique 3: Left Turn Overlaps}

Where an intersection has a median, some crossings to and from the median can run concurrently with a left turn phase as well as a through phase, an arrangement called an overlap. That can afford both longer WALK intervals and better pedestrian progression, thus reducing delay.

Consider the two-stage crossing of Beacon Street at St. Paul Street in Brookline, MA. Beacon Street has left turn phases, while St. Paul does not. Figure 5 shows the four possible ways of sequencing the Beacon Street left turn phases. 


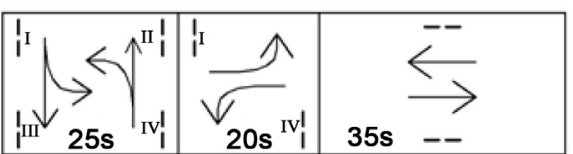

(a)

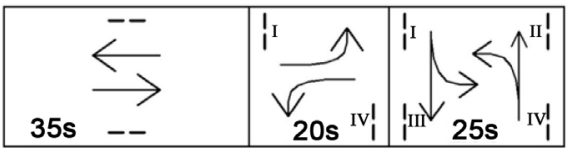

(c)

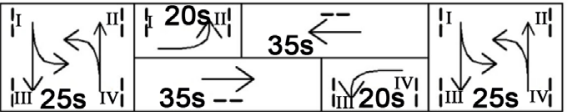

(e)

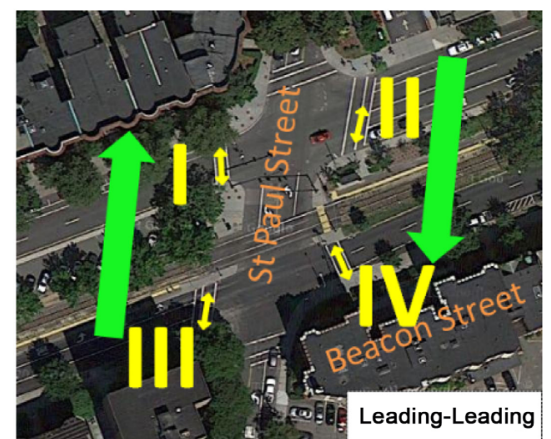

(b)

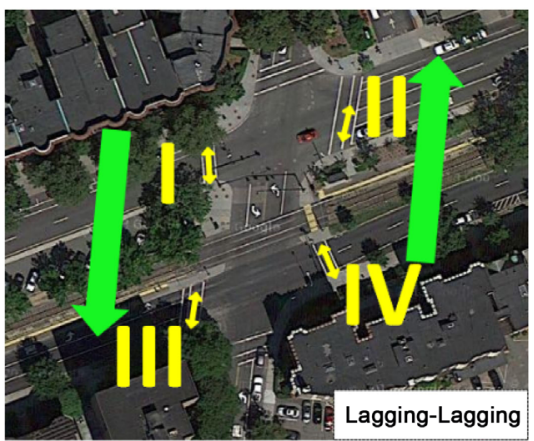

(d)

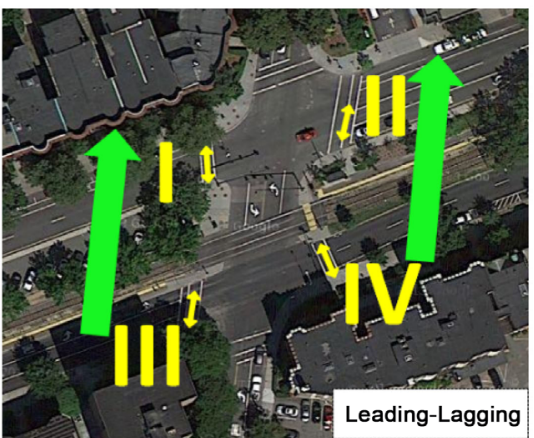

(f)

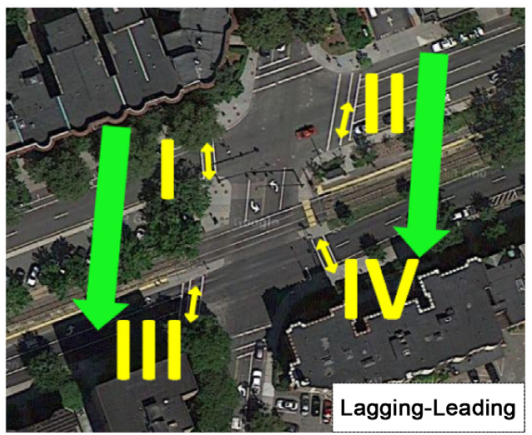

(h)

Figure 5. Sequencing left turn phases to enable single-stage crossings for some movements. (a) Lead-lead; (b) Movements with progression (lead-lead); (c) Lag-lag; (d) Movements with progression (lag-lag); (e) Lead-lag (Eastbound leads, westbound lags) (ring diagram shows $1^{1 / 2}$ cycles); (f) Movements with progression, lead-lag; (g) Lag-lead (Eastbound lags, westbound leads) (ring diagram shows $1^{1 / 2}$ cycles); (h) Movements with progression, lag-lead. 
Some sequences extend the WALK interval after the through phase, and some begin the WALK interval before the through phase, and therefore have different progression impacts. Figure 5 highlights which crossings get good progression with each sequence, assuming left turn overlaps are provided. Without overlaps (current operation), average pedestrian delay is $86.4 \mathrm{~s}$. With overlaps, if both lefts are leading (see parts (a) and (b) of Figure 5), crossing stages I and IV can be extended, giving both northbound and southbound pedestrians good progression-but only if they walk along the left side of the street. If both lefts are lagging ((c) and (d)), pedestrians again get good progression both northbound and southbound-but only if they walk along the right side of the street. Average delay is about $28 \mathrm{~s}$ for the side of the street with good progression versus $59 \mathrm{~s}$ for the other side. (An interesting question is whether to provide public information about which side is favored, or to let people discover it for themselves).

If one left turn leads and the other lags ((e) and (g)), all four crossing stages can be extended. Both sides of the street will have good progression, but in the same direction (either northbound or southbound); the other direction will not have good progression. However, because all the crossing stages are extended, average delay is only about $14 \mathrm{~s}$ for the favored direction and $38 \mathrm{~s}$ in the other direction, for an average of $26 \mathrm{~s}$. In spite of the fact that lead-lag systematically favors one direction of pedestrian movements, it still offers the lowest overall average pedestrian delay, unless pedestrians can be trained to walk on the side of the street with good progression.

Left turn overlaps are not currently provided at this intersection or at many like it. This may be because in order to use an overlap, when there is a pedestrian call, left turn phases must not be skipped and their minimum split might have to be greater than the minimum needed for vehicles. Would constraining the left turn phase so that it could be part of a pedestrian overlap significantly affect vehicular capacity and delay? At many intersections, including this one, the answer is no. During peak periods, the left turn phase is never skipped and nearly always runs to its maximum green, which is long enough for an overlap; and outside the peak, there is excess capacity, so that extra green time taken by the left turn phase would not significantly affect the through movement.

\section{Technique 4: Overlapping a Short All-Vehicles-Hold Phase}

A final technique for improving pedestrian progression at multistage crossings is a short phase in which no vehicular movements are allowed. The idea is not to create an exclusive pedestrian phase long enough to serve pedestrians by itself, but rather to use this "hold" phase to extend pedestrian phases in multiple directions in order to create good progression.

This technique can also be used to provide a single-stage crossing for bikes. Because bikes are faster than pedestrians, they need considerably less time for a single stage crossing. And where medians are not wide enough to safely hold a queue of bicycles, cyclists may need to have single stage crossings even where pedestrians make a two-stage crossing. This need is well understood in the Neth- 
erlands, where it is common to see the pedestrian phase begin (for pedestrians to cross to a median island) while the bike signal remains red until a time at which bikes can cross in a single stage, without stopping in the median.

This technique can be applied to the Riverway crossing that was described in Part 1 of this paper (see Figure 1). The crossing islands there are too narrow to hold queuing bikes, meaning that, in addition to having terrible service for pedestrians (average delay is $123 \mathrm{~s}$ ), there is no safe bicycle crossing.

A new traffic signal timing plan was developed for morning peak hour volumes with the existing cycle length (100 s), improving pedestrian progression by adding a $12 \mathrm{~s}$ phase in which all vehicular movements are held. As shown in Figure 6, that $12 \mathrm{~s}$ interval begins around time 55, and contributes to elongating all three crossing phases. Figure 6 shows the progression that results, with little waiting at the median islands; that can be contrasted with the poor progression evident in the existing timing (Figure 1). Also shown in Figure 6 is the average pedestrian delay, only $41 \mathrm{~s}$-a huge drop from the $123 \mathrm{~s}$ that prevails today. For bikes, the existing plan has no bike crossing at all (bikes must become pedestrians to cross), while the proposed plan offers bikes a single stage crossing with average delay $42 \mathrm{~s}$. At the same time, vehicular delay, evaluated using the microsimulation model VISSIM, increases by only $0.5 \mathrm{~s}$, from 34.8 to $35.3 \mathrm{~s}$. For more detail on the analysis of this intersection, please see the case study report [11].

Technique 5: Sequencing Phases for Cyclist Two-Stage Left Turn Progression

Limiting delay for two-stage cyclist left turns is a matter of having a short cycle and providing good progression between the through phases involved in the two-stage turn [10]. Where intersections have left turn phases, some phase sequences provide better progression for two-stage left turns than others.

Consider a junction of two streets $\mathrm{O}$ and D. Suppose the only left turns of concern are from $\mathrm{O}$ to $\mathrm{D}$, as might occur if the intersection is skewed. Good progression from $\mathrm{O}$ to $\mathrm{D}$ can be achieved by having $\mathrm{O}$ 's through movement immediately succeeded by D's through movement. That implies that if both streets have left turn phases, O's through movement should lag, while D's through movement leads. Looked at another way, O's left turn should lead while D's left turn lags.

Where all four left turns are of concern and there is a full set of left turn phases, there is no sequence offering good progression for all four left turns if bicycle crossings are unidirectional, as is the usual case.

However, if bicycle crossings are bidirectional, having one street's through movements lead while the other's lags allows all two-stage turns to be made with good progression, because every left turn can begin with a lagging through phase followed by a leading through phase. This is illustrated in Figure 7, where left turning cyclists have good progression if those approaching the intersection at $\mathrm{A}$ and $\mathrm{C}$ make their left turn by traveling counterclockwise and the those approaching at B and D make their left turn by traveling clockwise. 


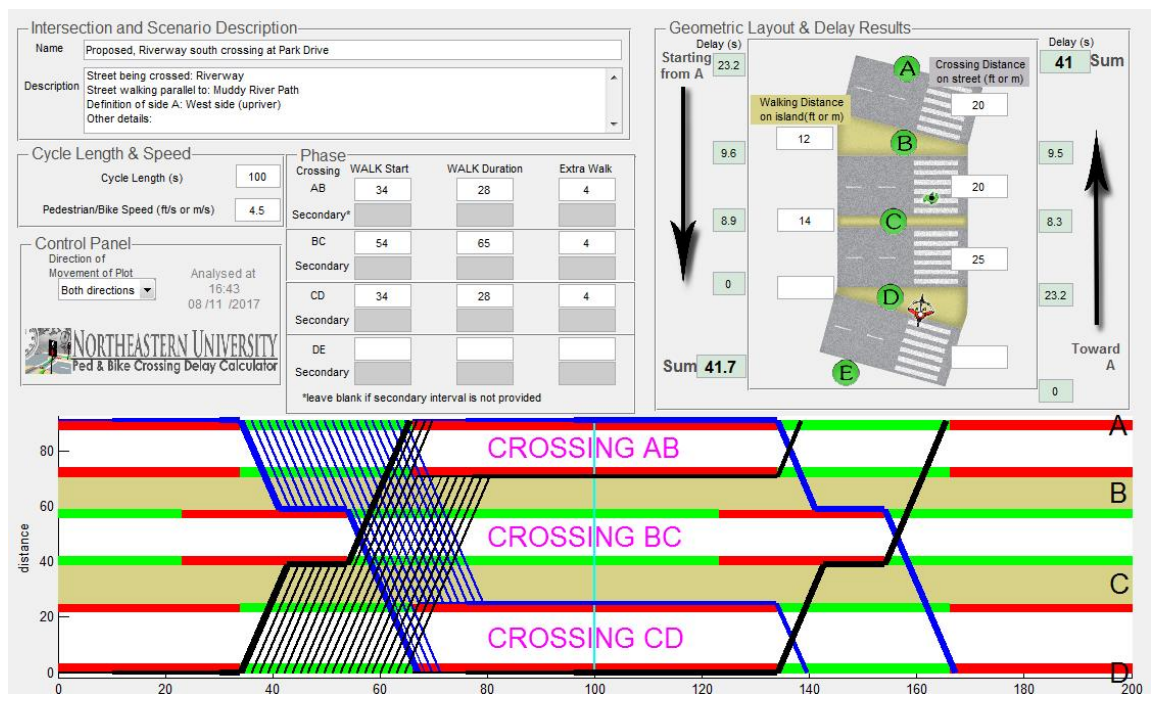

Figure 6. Proposed signal timing plan for the riverway crossing, with better pedestrian progression and a single stage bike crossing.

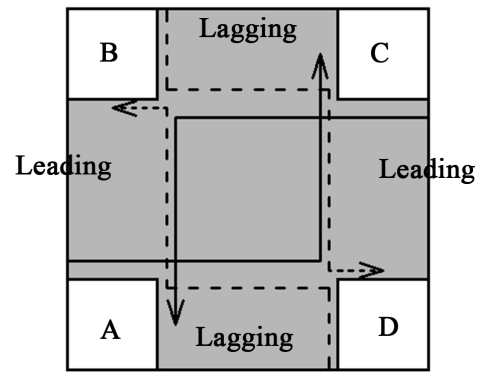

Figure 7. With bidirectional crossings and through phases that lead on one street and lag on the other, all four two-stage bicycle left turns get good progression (first leg lags, second leg leads).

\section{Conclusions}

The lack of existing methods for calculating pedestrian delay at multistage crossings means that signal timing plans with multistage crossings are sometimes developed that inadvertently lead to very long pedestrian delays because of the poor progression they offer from one stage to the next. We have demonstrated a simple numerical method with which multistage pedestrian delay can be readily calculated, even if there are multiple stages or multiple WALK intervals in a cycle, and also for diagonal crossings in which pedestrians can choose their path. This method can also be used to evaluate delay for cyclists making a two-stage left turn.

While multistage crossing delay can be very long where cycles are long and pedestrian progression is poor, numerous signal timing techniques can be used to create good pedestrian progression. In many cases, they can drastically improve service for pedestrians with little or no impact on vehicles. At one intersection with a 3 -stage crossing, we show how pedestrian delay can be reduced by more than $80 \mathrm{~s}$ while vehicular delay remains essentially unchanged. Phasing 
techniques were also described that can reduce delay for cyclist two-stage left turns, and provide for safe bicycle crossings where medians are too small to safely hold a cyclist queue.

\section{Conflicts of Interest}

The authors declare no conflicts of interest regarding the publication of this paper.

\section{References}

[1] FHWA (2009) Manual on Uniform Traffic Control Devices. Washington DC.

[2] National Association of City Transportation Officials (2011) Urban Bikeway Design Guide. National Association of City Transportation Officials, New York.

[3] National Research Council (2000) Highway Capacity Manual. TRB, National Research Council, Washington DC.

[4] Pretty, R. (1979) Delay to Pedestrians and Vehicles at Signalized Intersections. ITE Journal, 49, 20-23.

[5] Wang, X., Tian, Z.Z., Ohene, F. and Koonce, P.J.V. (2009) Pedestrian Delay Models at Signalized Intersections Considering Signal Phasing and Pedestrian Treatment Alternatives. Presented at 88th Annual Meeting of the Transportation Research Board, Washington DC.

[6] Wang, X. and Tian, Z. (2010) Pedestrian Delay at Signalized Intersections with a Two-Stage Crossing Design. Transportation Research Record, 2173, 133-138. https://doi.org/10.3141/2173-16

[7] Ma, W.J., Liu, Y., Xie, H.Z. and Yang, X.G. (2011) Multiobjective Optimization of SignalTimings for Two-Stage, Midblock Pedestrian Crosswalk. Transportation Research Record, 2264, 34-43. https://doi.org/10.3141/2264-05

[8] Furth, P.G. (2015) Northeastern University Ped \& Bike Crossing Delay Calculator. http://www.northeastern.edu/peter.furth/delaycalculator/

[9] Furth, P.G., Cesme, B. and Muller, T.H.J. (2009) Lost Time and Cycle Length for an Actuated Traffic Signal. Transportation Research Record, 2128, 152-160. https://doi.org/10.3141/2128-16

[10] Design Manual for Bicycle Traffic. CROW, Ede, Netherlands, 2007.

[11] Santos, M.A. and Furth, P.G. (2017) Reducing Pedestrian Delay at the Landmark Center Interchange. https://web.northeastern.edu/advancedtrafficcontrol/?p=406 